\title{
Healthcare provider beliefs about exercise and fatigue in people with multiple sclerosis
}

\author{
Catherine M. Smith, PhD; ${ }^{*}$ Leigh Anne Hale, PhD; ${ }^{1}$ Karin Olson, PhD, Distinguished Scholar; ${ }^{2}$ G. David Bax- \\ ter, PhD; ${ }^{1}$ Anthony G. Schneiders, PhD $^{1}$ \\ ${ }^{1}$ Centre for Health, Activity and Rehabilitation Research, University of Otago, Dunedin, New Zealand; ${ }^{2}$ Faculty of \\ Nursing, University of Alberta, Edmonton, Canada; and International Institute for Qualitative Methodology, Univer- \\ sity of Alberta, Edmonton, Canada
}

\begin{abstract}
This study describes the experiences of four groups of healthcare providers who facilitate exercise interventions for people with multiple sclerosis (MS)-related fatigue. Fatigue is a complex symptom frequently experienced by people with MS, yet it is poorly understood by clinicians and clinical researchers. Historically, clinicians have recommended less physical activity in order to limit fatigue; however, recent experimental studies suggest that regular exercise provides health benefits with little increase in fatigue. We used interpretive description methodology to guide data collection and analysis. Four groups of healthcare providers participated in either focus group discussions or individual interviews. Transcripts were analyzed for key meanings. Healthcare providers described their perceptions of the "nature of fatigue" and how this raised "professional challenges," specifically "barriers to implementation" of interventions, "stirring conflict" among interdisciplinary members, and "modifying roles." The nature of fatigue and professional challenges influenced clinician practice by "demanding creativity" with regard to exercise prescription and advice. Healthcare providers are encouraged to consider strategies of active listening and careful observation when providing individualized exercise programs for people with MS-related fatigue. In addition, recognition and understanding of the complex nature of fatigue by the interdisciplinary team might facilitate more positive exercise experiences for this population.
\end{abstract}

Key words: beliefs, exercise, experiences, fatigue, healthcare provider, interdisciplinary team, interpretive description, multiple sclerosis, physical activity, qualitative research.

\section{INTRODUCTION}

Fatigue is a complex symptom frequently experienced by people with multiple sclerosis (MS) yet poorly understood by clinicians and clinical researchers [1]. Historically, healthcare providers (HCPs) have recommended less physical activity as one energy-conservation strategy to limit fatigue symptoms; however, recent experimental studies suggest that regular exercise provides health benefits, such as improved strength, cardiovascular fitness, and quality of life for some people with MS, with little associated significant changes in fatigue [2]. Despite these positive results, fatigue is often reported as a barrier to exercise participation [3-5], and little is known about the way in which HCPs prescribe or advise exercise for people with MS with this symptom. In one qualitative study, participants with MSrelated fatigue undertaking a progressive resistance exercise program felt uncomfortable when exercise techniques

\footnotetext{
Abbreviations: $\mathrm{HCP}=$ healthcare provider, $\mathrm{MS}=$ multiple sclerosis, OT = occupational therapist, $\mathrm{PT}=$ physiotherapist/ physical therapist.

*Address all correspondence to Catherine $\mathrm{M}$. Smith, PhD; Centre for Health, Activity and Rehabilitation Research, School of Physiotherapy, University of Otago, PO Box 56, Dunedin 9054, New Zealand; +64-3-479-7473; fax: +64-3479-8414. Email: cath.smith@otago.ac.nz http://dx.doi.org/10.1682/JRRD.2012.01.0012
} 
and equipment operation were not explained satisfactorily; when exercise rules were too rigid; and when instructors failed to include strategies that were perceived as important, such as stretching or warm-ups [6]. In addition, people with MS who choose to exercise have described feelings of confusion and frustration when HCPs offered advice regarding exercise and energy management that conflicted with participants' own experiences [7]. More recently, Smith et al. (2011) found that persons who experienced MS-related fatigue were frustrated when HCPs failed to recognize the importance of individual exercise limits, often learned by the people with MS through trial and error rather than advice from a health professional [7]. In this study, participants welcomed HCP advice and monitoring of exercise for people with MS-related fatigue; however, they also expressed the desire for greater and more consistent understanding regarding individual experiences and choice of exercise [8].

The authors of a postal survey of 318 people with MS that examined health preferences and access to HCPs found that 41 percent of participants wanted more advice from HCPs about activities such as exercise [9]. The poorly understood nature of MS-related fatigue might pose a barrier to confident and consistent advice from HCPs regarding recommendation of exercise as a health-promoting intervention for people with MS. Therefore, the purpose of this study was to explore, describe, and interpret the experiences of HCPs who provide interventions and advice for people with MS-related fatigue in order to identify possible strategies that might assist with this process.

\section{METHODS}

\section{Design}

Interpretive description was used to guide data collection, analysis, and presentation of results [10]. A strength of this design is that it was developed by Thorne et al. to explicitly link the interpretation of the description resulting from the data to clinical practice [11], and hence, it was well suited to the research question for this study. In this design, interviews and focus groups are used to collect information from participants about their experiences in relation to the research question. These data are then analyzed line by line to identify broad themes supported with example quotes from the data. These themes are used to construct a detailed description of the experience of participants, which is then discussed in detail from a clinical perspective. A second strength of this design is that Thorne provides a detailed description of the process for ensuring rigor, which is carefully rooted in clinical practice [10]. Interpretive description was originally developed for use in nursing, but in the study described in this article, the results are discussed from the standpoint of several health professions.

\section{Sample and Setting}

Interpretive description recommends that the method of data collection should be chosen in order to best represent the research question [10]. In our study, we wanted to explore perceptions, opinions, and beliefs about exercise as an intervention for MS-related fatigue. We therefore chose to collect data via recorded, transcribed interviews with HCPs who had provided direct interventions, including strategies, advice, counseling, exercise prescription, and pharmaceutical prescription, for people with MS within the last 2 years. We purposefully sampled four HCP groups that had been named by participants in a previous study that explored fatigue and exercise experiences in people with MS: consultant neurologists, physiotherapists/physical therapists (PTs), occupational therapists (OTs), and MS Society support workers [7]. We distributed advertising flyers at regional PT and OT special interest meetings and a locally held national training day for MS Society field officers and sent individual letters of invitation to local consultant neurologists. Detailed information sheets were either e-mailed or posted to HCPs who contacted the author expressing interest in study participation. Eight PTs, four OTs, three MS Society support workers, and three neurologists agreed to participate in the study. Two PTs and one OT withdrew before the informed consent stage of the study because of time conflicts, leaving a total of 15 participants.

\section{Data Collection and Analysis}

On receipt of informed consent, we collected data via four methods. Local PTs and OTs participated in focus group discussions, PTs from other regions of New Zealand and MS Society support workers were individually interviewed via telephone, and consultant neurologists were individually interviewed face to face. Interview and focus group questions were guided by an open-ended question schedule and generally began with a contextual question such as "Could you tell me a little bit about your involvement with people with MS?” Examples of other questions from the guide included "How do you think exercise fits 
with fatigue in people with MS?," "How do you advise/ counsel or treat a patient with MS?," and "How would you describe your role in the management of fatigue for people with MS?” Because the project was inductive in nature, precise questions were not determined in advance and depended on individual experiences and the way the interview and focus groups developed. All audio recordings were transcribed verbatim. Field notes recording postinterview comments, observations, and author reflections were recorded during and following interviews and focus groups. During focus group discussions, in order to maximize the range of participant opinion, the facilitator noted whether any individual expressed opinions that differed from the majority of the group or if any participant appeared reluctant to contribute to the discussion. One participant from a focus group was contacted and offered a follow-up individual interview in order to more fully discuss the participant's opinions and beliefs.

We checked transcripts for accuracy against recordings and transferred the checked transcript into NVivo VIII software (QSR International Pty Ltd; Doncaster, Victoria, Australia). Any inconsistencies found between recording and transcripts were corrected by the primary author. The primary author read all transcripts several times and placed codes pertaining to the research question into preliminary categories in NVivo tree nodes. To strengthen the rigor of our analysis, we followed a four-step process:

1. Following preliminary identification of emerging codes and themes by the primary author, clean transcript hard copies were presented to a coinvestigator for analysischecking purposes. The coinvestigator identified codes from the data that were then cross-referenced with the primary author's preliminary grouping of codes into themes.

2. The primary author refined the analysis, adding key quotes to illustrate themes.

3. This preliminary analysis was given to an HCP independent of both study participation and geographical region of study participants for reflection and comment.

4. Following further reflection and exploration of both raw data and analysis, the primary author refined the written results.

These results were distributed to all 15 study participants for comment. Eight participants returned comments, which were synthesized into the final analysis. Because several study participants were from the same region of New Zealand, we decided to anonymize name and profession by identifying participant quotes with a number only.

\section{RESULTS}

Fifteen HCPs participated in this study: six PTs, three OTs, three MS Society support workers, and three consultant neurologists. Our participants all described regular contact with and provision of active advice/ intervention for people with MS over a 2-year period and, therefore, could be considered experienced practitioners in this field. However, the duration and frequency of contact varied among participants according to their role. For example, a consultant neurologist might see people with MS on most days of the week but would only see the same patient once or twice per year, whereas an MS Society support worker might see the same people each week for several years. Ten participants were from a single regional area; five participants were from other regions of New Zealand. We identified three interrelated themes from the data: the "nature of fatigue" resulted in "professional challenges," which led to "demanding creativity." We identified three subthemes that contributed to the professional challenges theme: "barriers to implementation," "stirring conflict," and "modifying roles" and two subthemes that contributed to the demanding creativity theme: "challenging science" and "mind-body." These themes and subthemes are illustrated in the Figure.

\section{Nature of Fatigue}

HCP participants described fatigue as one of the major symptoms experienced by their clients, yet the most difficult to address. Difficulties presented by fatigue included characteristics of unpredictability, invisibility, and individuality and was consequently life altering for people with MS. MS-related fatigue was perceived as having very little scientific explanation and often seemed inextricably linked to other MS-related impairments such as depression, poor balance, and cognitive difficulties. These characteristics resulted in MS-related fatigue being a difficult phenomenon for HCPs to conceptualize, describe, and measure:

I suppose it's one of those words [fatigue] we probably use and then if someone puts us on the spot asking us to say exactly what we mean by it, we find it very, very sort of difficult. It's a very subjective response and it is different for each person. (Participant 5)

Participants described the complexity of fatigue with reference to two distinct etiological factors: physiological processes associated within physical inactivity, such as 


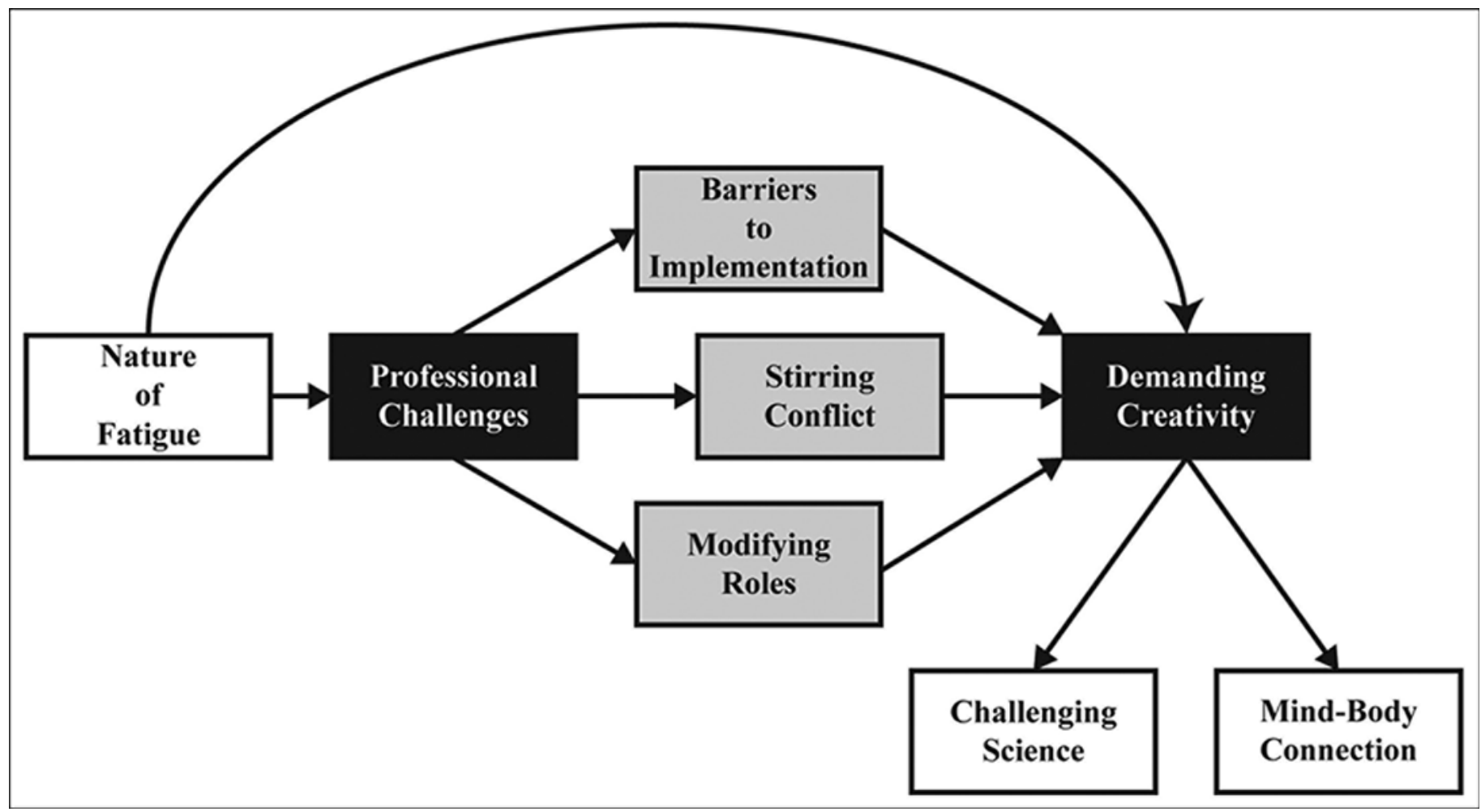

Figure.

How multiple sclerosis-related fatigue influences healthcare provider opinions of exercise as intervention for fatigue.

cardiovascular deconditioning and weight gain, and pathological factors, such as increased central nervous system excitation, which gave rise to central or "neurogenic fatigue.” In the absence of scientific explanation, participants seemed to identify strongly with one approach that held meaning for them, frequently based on factors such as personal or working experiences. Some participants believed that the invisibility of fatigue resulted in complex behavioral and psychosocial implications for their clients; for example, the perception that people with MS are lazy or manipulate for gain might result in clients being reluctant to divulge information relating to fatigue experiences:

The classic thing they'll say is "I thought I was going crazy, I thought I was lazy.” (Participant 12)

The unpredictability of fatigue meant that, although always present, the ways in which fatigue was experienced by clients varied both between and within individuals:

. . . There's a lot of descriptions for fatigue. When you go around ten different patients they will give you a different description. If nobody knows what fatigue is you can quite understand why there's a lot of differing beliefs about it .... (Participant 2)

Participants also described fatigue as affecting aspects of clients' lives including employment, relationships, personal expectations, and ability to follow health-related recommendations.

Fatigue was perceived as time consuming in terms of HCP consultation and management, for example, involving extended and/or multiple client interviews. The neurologists in this study acknowledged that there was little time during the course of a hospital appointment to explore fatigue experiences fully, and consequently, they referred clients on to other HCPs for fatigue management.

\section{Professional Challenges}

Difficulties with conceptualization, verbalization, and measurement of MS-related fatigue resulted in three professional challenges: barriers to implementation, stirring conflict, and modifying roles. 


\section{Barriers to Implementation}

Barriers to the implementation of interventions, including exercise, for people with MS-related fatigue included personal, cognitive, financial, environmental, social, service provision, and knowledge factors. HCP participants believed that the invisible nature of fatigue often led to clients feeling as if they were "different," even though these differences were not outwardly apparent. These feelings resulted in a reluctance to exercise publicly, for example, in a gym or at a swimming pool. In addition, one participant felt that people with MS-related fatigue were more likely to seek exercise activities independently rather than through an HCP as a result of invisible impairments.

Lack of motivation and lack of interest in exercise were attributed to two different sources in people with MS; however, it was difficult for the HCP participants to differentiate between these sources. Firstly, these factors were attributed to the personal interests, priorities, and positive or negative attitudes of the individual client. Secondly, these same factors were attributed to personality, motivational, and cognitive changes associated with the process of MS. Financial limitations and transport difficulties were also discussed by participants; it was noted that under the jurisdiction of some regional MS societies, gym memberships, exercise classes, and transport are funded, and while these barriers are removed, motivational barriers often remained. Several participants recognized that clients needed to enjoy exercise activities in order to remain motivated. HCP participants recognized the value of social support for people with MS-related fatigue seeking interventions such as exercise. Support networks included amenable employers, family support, and on-going HCP input.

Lack of scientific knowledge with reference to possible interventions for fatigue created barriers for some participants, who felt that anything offered for the management of MS-related fatigue could best be described as "tinkering" with the symptom in the absence of any evidence base. Participants also felt that a lack of knowledge existed between healthcare team members with regard to their role within the interdisciplinary team. In addition, participants described a lack of knowledge in people with MS of "what's out there" regarding services and assistance available both in the healthcare as well as the community and leisure industries. Service barriers such as limited ability to offer suitable appointment times, inappropriate timing of referrals, and time restrictions within appointments to discuss fatigue were discussed.

\section{Stirring Conflict}

Several areas of conflict were described by participants in this study, including interprofessional conflict, provider versus client conflict, knowledge conflicts, and client motivational conflicts. There was a perception among some participants that one profession "owned the word fatigue" (Participant 15), which led one participant to stop using the word "fatigue" and instead refer to levels of "cardiovascular fitness." Exercise as a concept also created a dilemma and was considered by several participants as potentially beneficial or harmful depending on context and experience of the individual. The perceived nature of fatigue led to interprofessional conflict regarding "ownership" of fatigue, particularly in PT and OT participants in the study. Subsequently, this resulted in further conflict in regard to fatigue management approaches. For example, PTs perceived their management style as introducing graded levels of exercise in order to increase fitness levels and thereby reduce fatigue attributed to poor cardiovascular endurance. OTs perceived their management style as prioritizing activities in the context of daily living, which sometimes meant reducing the amount of exercise undertaken by a client in order to conserve or create energy for more meaningful activities.

So, because again people's different belief systems, philosophies, training, you end up having a bit of a battle whenever you start discussing somebody's fatigue because another profession just doesn't believe that we're qualified to be discussing it. (Participant 15)

Interprofessional conflict was perceived by HCP participants as having negative influences on clients. For example, one participant expressed concern that patients could easily sense interdisciplinary conflict at team meetings:

... That's why I think it's hard on patients because it's so confusing for them and they're not stupid they can pick up that little bit of "hold on I'm getting a slightly different message from these two." (Participant 6)

A perceived dearth of knowledge regarding the physiological nature of fatigue and interventions for fatigue was problematic for study participants. In the absence of scientific knowledge, participants in this study discussed how unproven theories and "gurus" might influence practice. Some participants relied more on personal experience when approaching fatigue management and offered exercise and nutrition guidelines based on their own 
"tried and tested" health behaviors. It must be noted that both participants and an independent analysis reviewer commented that interprofessional conflict might depend on individual personality and organizational differences between healthcare settings.

Conflict also occurred when clients and HCPs had contrasting perceptions of priority; for example, when a client prioritized his or her role caring for family, yet the health professional felt the client should prioritize his or her own health. In addition, participants described occasions where another HCP suggested a fatigue reducing strategy that did not make sense to the client. As one participant observed:

So that [HCP] recommendation around sort of ... shortening hours of work just wasn't something she was going to consider because for her that's not where the fatigue was the issue, it was more the weekends and the running around with little kids when her husband worked on a Saturday so she had the children. Like I say she found that more tiring than a day at work because she was sitting behind an office desk. (Participant 4)

\section{Modifying Roles}

The professional challenges encountered, as a consequence of the nature of fatigue, included difficulties with modifying roles, including blurring of professional boundaries, introduction of nontraditional roles, restricted longterm roles, and lack of flexibility to move between roles. Roles that featured strongly across all participants were those of education, advice, empowerment, confidence building, encouragement, and support.

Blurring of professional boundaries seemed to be related to the complex nature of MS-related fatigue, changes in roles over time, and conflicts regarding perceived "ownership" of roles. Some participants felt their roles were changing; for example, one participant explained that the role of exercise had changed from maintenance of existing movement and functional status to focusing on improving cardiovascular function, muscle strength, quality of life, and long-term good health. Some participants perceived their roles to be undervalued and, at times, poorly understood or undermined by other members of the healthcare team.

The complex nature of fatigue appeared to result in the development of nontraditional roles. For example, one participant described employing "detective work" in order to assess the complex and individual nature of fatigue. Barriers to implementation, such as low motivation, experienced in people with MS-related fatigue also led to a perceived necessity to become a "creative salesperson”; for example, one participant describes:

So how I try to sell . . . exercise to a lady recently was ... you know you would invest in a pension, you invest in all these things for later on in life when you retire but the one thing we do know from the evidence ... is that if you exercise regularly then this is going to stave off a lot of the other nasties. (Participant 11)

Some HCP participants had begun to introduce nontraditional interventions including yoga and meditation into their role. These participants, through observation and active listening, had a perceived awareness of a "mind-body" connection with regard to fatigue that could be manipulated beneficially with these practices. Several participants in this study perceived ongoing support to be an important part of their role and, as a consequence, experienced frustration when their ability to provide this support was restricted by service arrangements, lack of referral, and time restrictions. Participants who were able to monitor, review, observe, and interact with clients over long periods of time seemed to experience greater satisfaction in their role. This, in part, appeared related to optimal timing concerning client readiness to change coinciding with the HCP being ready and able to intervene. This level of support enabled them to step in and "fine tune" interventions when required. Client empowerment was regarded by several participants as a key part of their role and included, specifically, increasing levels of control, self-efficacy, choice, body awareness, and self-monitoring in clients:

. . . being encouraged to respect the fact that it's their bodies and they know what they're feeling is truly what they are feeling, and through that having some empowerment that they can make decisions, the right decisions for that point in time. (Participant 7)

Many participants in the study described their role in helping individuals manage fatigue as a multiple role, one that demanded flexibility between roles, depending on the individual experience of fatigue and the immediate needs of the client. Both the nature of fatigue and the professional challenges created by this symptom influenced how HCPs provided exercise interventions for clients by demanding creativity. This theme and two subthemes, 
challenging science and the mind-body connection, are described in the next section.

\section{Demanding Creativity}

In the perceived absence of scientific evidence for the physiological basis of fatigue and to support the effectiveness of possible interventions for fatigue, HCP participants in this study reported that they used a variety of creative strategies to inform and shape their practice. These strategies were labeled "creative" because they relied on personal experiences; working experience; trial and error; and observing, listening to, and identifying with the client.

Experiences that shaped creative practice included personal experience of illness-related fatigue, personal health benefits including reduced fatigue from exercise participation, and observation of health behaviors in relatives or friends with illness-related fatigue. Many participants felt that understanding gained from extended working experience helped to guide their practice. This included other illness or injury, such as working in the field of chronic pain. Participants were able to recognize indicators of success, particularly when clients experienced "Aha moments," alternatively described as "Light bulb moments," "Acceptance," and "Ready for change”: “. . . but once that light bulb goes on it's just brilliant because it's like wahey” (Participant 2). Recognizing these moments seemed to play a pivotal role in shaping management of MS-related fatigue, and participants felt that at these moments, their role changed. For example, with reference to exercise interventions, one PT felt that until a client reached an "Aha moment," their role was to inform, advise, and provide ongoing monitoring. In contrast, once a client had reached an "Aha moment," the PT was also able to offer exercise alternatives, tailor programs individually, and monitor functional and fitness progress. Participants felt that these moments indicated that clients had reached a point of acceptance where they were willing to acknowledge the presence of MS and make positive changes to optimize their health and quality of life.

The strategy of trial and error was linked to all aspects of management practice for MS-related fatigue and seemed to play an integral role in the process of growing sense of control, self-efficacy, and adherence for clients. In addition, trial and error seemed linked to the ability of participants to fine tune interventions for clients.

But really I guess when they're taking control of it because then you know that it's going to happen and it's not this whole "I'm talking to you about this because I know it's good but at the end of the day I really know you don't want to have a bar of it.” (Participant 6)

An additional strategy used by participants in this study was a process of active listening and careful observation. This process appeared to involve sophisticated listening skills that identified implicit as well as explicit cues regarding client priorities, goals, and problems. This process required strategies that ensured the client was aware that his or her concerns were heard and included the use of imagery to try to understand and/or identify with the fatigue experience; for example, one participant compared MS-related fatigue to the fatigue experienced during menstruation and another to posttraumatic stress syndrome. Other strategies used by participants included listening for concerns that were repeated more than once by clients during an interview, discussing those concerns in the context of the intervention session, and using analogies to assist clients in verbalizing their fatigue experiences. Additional strategies such as fatigue diaries and the development of comprehensive fatigue assessment tools were described by one participant as being useful strategies to make the invisible nature of fatigue more visible to the client, the family, and the healthcare team.

Most participants used one or more of these strategies to guide their practice; however, those using active listening and observation felt it necessary at times to ignore current theories about both MS-related fatigue and exercise practices, shaping their practice according to observed success and feedback from clients. This process demanded creativity, courage, and confidence, because this approach might be considered soft, alternative, or "touchy-feely" by other members of their own profession and the wider healthcare community. This process involved two strategies: challenging science and recognizing the mind-body connection. These strategies had several implications for exercise recommendations for clients with MS-related fatigue.

\section{Challenging Science}

Participants recognized that their clients were able to achieve better perceived health and functional gains with far less exercise frequency than was recommended at the time in scientific medical literature and that this could lead to less fatigue in their clients with MS:

I know that we talk about you have to do things three times a week and we set these big goals for people and we think they need a lot of input, but for this lady, once a week was actually enough to 
achieve something quite impressive.

(Participant 14)

Several participants felt that functional improvements could be made by incorporating exercise into everyday life because "at the end of the day activity is exercise” (Participant 3).

\section{Mind-Body Connection}

Several participants in this study who recognized a mind-body connection believed that a positive attitude could influence adherence to an exercise program and, furthermore, that exercise participation could help to strengthen this positive attitude and increase motivation. Participants who recognized this mind-body connection also perceived a link between mental and physical fatigue and incorporated strategies such as meditation, relaxation, and body awareness into exercise programs. The mindbody connection was perceived as more than belief based and seemed to center on increasing movement efficiency and reducing energy expenditure through increased body awareness and mental relaxation. For example, one participant who taught Pilates to a group of people with MS described the influences of improving posture on mood:

So I think of it that perhaps with Pilates, the exercise can perhaps affect the way they are feeling by increasing you know shoulders up, and head up, perhaps you can reverse it the other way and make them psychologically feel a little bit better with their bodies being held better. (Participant 7)

\section{DISCUSSION}

This study explored the perceptions, beliefs, and opinions of four groups of HCPs: consultant neurologists, PTs, OTs, and MS Society support workers who provide interventions and advice for people with MS-related fatigue.

The four groups of HCPs in this study were selected on the basis of being named in participant narratives from a previous study [7]. The frequency of contact with these groups of HCPs was reflected in a UK-based postal survey conducted by Somerset et al. (2001), which indicated that the four HCP groups in our study were ranked in the six most frequently consulted healthcare professionals by 316 people with MS [9]. In this survey, general practitioners and consultant neurologists were, respectively, the first and second most frequently consulted HCPs, yet consultant neurologists in our study felt they had little time to discuss complex symptoms, such as fatigue, with patients and relied more on referral to specialist MS nurses, PTs, and OTs for this role. Because several participants in our study felt that fatigue could be managed more efficiently in the long term with early intervention, it may be useful for HCPs, particularly consultant neurologists and general practitioners, to consider early referral of individuals experiencing fatigue to a team member who may have more time and resources to assess, monitor, and provide ongoing support for the management of this symptom. We did not invite general practitioners to participate in this study because they had not been identified by participants in our previous study; however, given the results of Somerset et al.'s survey [9], we recommend that future studies explore strategies used by general practitioners when making recommendations for people with MS-related fatigue.

The subtheme of perceived interprofessional conflict was strongly represented by the data in this study, particularly between PTs and OTs, who each demonstrated different management styles with regard to fatigue minimization. These differing management styles appeared to cause tension between the two professions, an occurrence that many felt could influence clients negatively, particularly when client-centered meetings were held with client and HCP both present. Participants observed a perceived conflict between achieving a successful client-centered team strategy and an individual desire to maintain professional identity. Wanting to maintain a sense of professional identity could also be related to the subtheme of modifying roles. Previous research has identified that role modification, specifically role ambiguity, role erosion, and role extension, influences professional identity [12]. Several participants identified a functional interdisciplinary team as a key attribute in the successful delivery of energy management interventions for people with MS. A survey that examined health professional responses to both MS and motor neurone disease described difficulties encountered by HCPs, including resource issues, the ability to remain positive in the face of progressive disability, and interdisciplinary team conflicts [13]. The same study identified strong, supportive teamwork as being one of the most useful strategies when caring for clients with both MS and motor neurone disease [13]. Addressing interdisciplinary team dynamics may prove a useful direction for future research regarding delivery of interventions for individuals with MS-related fatigue. We suggest that multidisciplinary collaboration with regard to testing and development of intervention 
strategies for MS-related fatigue might be one way of increasing interdisciplinary understanding of this complex symptom.

Some HCP participants suspected that the invisible nature of fatigue might pose a barrier to engagement in exercise interventions as a result of fatigue being largely ignored by the healthcare community. White et al. (2008) recommended regular screening for all invisible symptoms of MS, including depression, pain, and fatigue, because their study found that invisible symptoms were more predictive of health distress than visible symptoms [14].

As discussed, the complex and poorly understood nature of fatigue led to feelings of interdisciplinary conflict and was perceived as creating a barrier to help-seeking in the community. It would seem prudent to suggest that an educational model regarding the multidimensional nature of MS-related fatigue might be developed and introduced to all health science students, practicing healthcare professionals, and advocacy organizations. Such a model might, for example, recognize that fatigue due to deconditioning is only one factor among several psychological, psychosocial, pharmaceutical, and pathophysiological contributors that make up a person's self-reported experience of fatigue [15]. It might be useful in future studies to determine how understanding of MS-related fatigue is taught/learned in different educational environments.

With little evidence regarding physiological basis, several participants in this study felt it necessary to draw on creative strategies in order to deliver more effective interventions, such as exercise, to people with MS-related fatigue. These strategies seemed to be based on careful observation of client performance and verbal feedback, rather than reliance on established exercise recommendations. Exercise recommendations before 2011 advocated participation in aerobic and strengthening activities several times a week for maintenance of cardiovascular health in the general population [16]. A recent consensus report by the American College of Sports Medicine suggests that exercise participation might continue to provide health benefits if performed less than recommended in prior guidelines [17]. At the time we interviewed HCPs, these latest recommendations had not been published; therefore, it is exciting to discover that clinician experiences reflected these new recommendations before their publication. Previous studies that examined the effects of exercising once a week in people with MS reported improvements in fatigue, balance, perceived walking ability, and measured walking endurance [18-19]. It is possible, therefore, that exercising once a week may lead to improvements in both perceived functional ability and measured function and balance. Further research is necessary to clarify whether these changes are perceived and/or physiological in nature. In light of recent exercise recommendations, HCPs and individuals with MS who wish to exercise might feel more confident that exercise activities performed less frequently could still contribute to better health. These recent exercise recommendations also support the practice of yoga and tai chi, activities recognized by our participants as forms of exercise, which highlight the usefulness of a mind-body connection [16]. For example, some participants found yoga useful as a fatigue-minimizing activity. A recent exercise consensus report has discussed the potential benefits of mind-body activities such as tai chi, particularly with reference to the positive effects this type of activity can have on perceived quality of life in the older adult [17]; however, Wahbeh et al. (2008) also concluded that evidence was insufficient to recommend mind-body interventions in neurological populations primarily because of insufficiently powered studies [20].

Our HCP participants described strategies that they found helpful when advising or prescribing exercise activities. These strategies included active listening, careful observation, and recognition that self-reported fatigue was a highly individual experience and, furthermore, that empowerment, confidence building, encouragement, and support were important aspects of their role. Previous studies that explored the experiences of people with MS when communicating with HCPs reflect our findings. For example, Thorne et al. (2004) described how important it was for patients to feel that their experiences of MS were acknowledged and affirmed by healthcare professionals [21]. Several studies that explored exercise experiences in people with MS recommended the development of an individual and tailored approached to both exercise and other areas of MS management [6,22-23]. We suggest that a successful individual and tailored approach may depend on the helpful strategies described by our HCP participants.

We used interpretive description to guide our research methods, many of which are used in other qualitative approaches such as grounded theory, phenomenology, and ethnography [10]. Interpretive description differs from these traditional approaches in that, while it uses similar methods, it explicitly links the description from the data to clinical practice. More traditional approaches generate theory and knowledge that is not 
designed to be used in an immediate practical sense. Arguably, this approach might result in more efficient clinical translation; however, we acknowledge that results from one qualitative study cannot be generalized to other contexts. Thorne (2008) strongly recommends follow-up interviews in order to clarify data meaning [10]. In this study we reinterviewed only one participant, because our initial interviews yielded a large amount of richly described data. We acknowledge that with more time, further interviews might have allowed greater refinement of our analysis. A further limitation of our study was the brevity of our sample description. We acknowledge that this minimal description might compromise the reader's contextual understanding of the result. The majority of our participants were from one region of New Zealand, and in order to maintain participant anonymity as far as possible, we decided not to attribute professional role or other descriptions when using quotes in our analysis. Future studies might consider sampling HCPs across a wider geographical area, thus increasing ability to describe the sample without compromising anonymity.

\section{CONCLUSIONS}

The complex and poorly understood nature of fatigue might result in professional challenges, particularly interdisciplinary conflict and creative practice. Interdisciplinary conflict might be minimized through frank reflection and discussion by the HCP interdisciplinary team, particularly in regard to identifying and addressing autonomous professional needs while maintaining the integrity of a client-centered approach. Furthermore, multidisciplinary collaboration in future research, combined with a multidisciplinary approach to undergraduate education, might increase knowledge and understanding of interventions for MS-related fatigue. HCPs are asked to consider active listening and careful observation as strategies that might optimize creative fatigue management strategies when prescribing interventions such as exercise and further minimize possible conflict. With specific regard to exercise strategies that might minimize fatigue, clinicians should consider exploration of strategies that incorporate exercise into daily activities; exercise performed once a week; and exercise activities that are perceived to include a mind-body focus, such as yoga and Pilates.

\section{ACKNOWLEDGMENTS}

\section{Author Contributions:}

Study concept and design: C. M. Smith, K. Olson.

Data acquisition: C. M. Smith.

Analysis and interpretation of data: C. M. Smith, L. A. Hale, K. Olson. Drafting of manuscript: C. M. Smith.

Obtained funding for project: C. M. Smith.

Critical revision of manuscript for important intellectual content:

L. A. Hale, K. Olson, G. D. Baxter, A. G. Schneiders.

Study supervision: L. A. Hale, K. Olson, G. D. Baxter, A. G. Schneiders.

Financial Disclosures: The authors have declared that no competing interests exist.

Funding/Support: This material was based on work supported by a Physiotherapy New Zealand Neurology Group Research Grant and University of Otago PhD Scholarship.

Additional Contributions: The authors would like to thank all the participants for sharing their experiences and Jenni Hammington for her thoughtful commentary on the preliminary analysis.

Institutional Review: Ethical approval for this study was granted by the New Zealand Lower South Regional Ethics Committee (approval number: LRS/07/12/046).

Participant Follow-Up: The authors will endeavour to contact all participants in this study to inform them of publication; however, some participants are no longer available through contact information used during the data collection period.

\section{REFERENCES}

1. Smith C. Fatigue in people with multiple sclerosis: challenges in evaluation of interventions. Phys Ther Rev. 2006; 11(2):75-76. http://dx.doi.org/10.1179/108331906X99029

2. Rietberg MB, Brooks D, Uitdehaag BM, Kwakkel G. Exercise therapy for multiple sclerosis. Cochrane Database Syst Rev. 2005;1(1):CD003980. [PMID:15674920]

3. Borkoles E, Nicholls A, Bell K, Butterly R, RC, Polman. The lived experiences of people diagnosed with multiple sclerosis in relation to exercise. Psychol Health. 2008;23(4):427-41. http://dx.doi.org/10.1080/14768320701205309

4. Stroud N, Minahan C, Sabapathy S. The perceived benefits and barriers to exercise participation in persons with multiple sclerosis. Disabil Rehabil. 2009;31(26):2216-22. [PMID:19903131] http://dx.doi.org/10.3109/09638280902980928

5. Stuifbergen AK, Roberts GJ. Health promotion practices of women with multiple sclerosis. Arch Phys Med Rehabil. 1997;78(12 Suppl 5):S3-9. [PMID:9422001] http://dx.doi.org/10.1016/S0003-9993(97)90215-X

6. Dodd KJ, Taylor NF, Denisenko S, Prasad D. A qualitative analysis of a progressive resistance exercise programme for people with multiple sclerosis. Disabil Rehabil. 2006;28(18): 1127-34. [PMID:16966233] http://dx.doi.org/10.1080/09638280500531842 
7. Smith C, Hale L, Olson K, Schneiders AG. How does exercise influence fatigue in people with multiple sclerosis? Disabil Rehabil. 2009;31(9):685-92. [PMID:18841515] http://dx.doi.org/10.1080/09638280802273473

8. Smith C, Olson K, Hale LA, Baxter D, Schneiders AG. How does fatigue influence community-based exercise participation in people with multiple sclerosis? Disabil Rehabil. 2011;33(23-24):2362-71. [PMID:21504405]

9. Somerset M, Campbell R, Sharp DJ, Peters TJ. What do people with MS want and expect from health-care services? Health Expect. 2001;4(1):29-37. [PMID:11286597] http://dx.doi.org/10.1046/j.1369-6513.2001.00111.x

10. Thorne S. Interpretive description. 1st ed. Walnut Creek (CA): Left Coast Press Inc; 2008.

11. Thorne S, Kirkham SR, MacDonald-Emes J. Interpretive description: a noncategorical qualitative alternative for developing nursing knowledge. Res Nurs Health. 1997;20(2): 169-77. [PMID:9100747]

http://dx.doi.org/10.1002/(SICI)1098240X(199704)20:2<169::AID-NUR9>3.0.CO;2-I

12. Hobart JC, Riazi A, Lamping DL, Fitzpatrick R, Thompson AJ. Measuring the impact of MS on walking ability: the 12-Item MS Walking Scale (MSWS-12). Neurology. 2003; 60(1):31-36. [PMID:12525714] http://dx.doi.org/10.1212/WNL.60.1.31

13. Carter H, McKenna C, MacLeod R, Green R. Health professionals' responses to multiple sclerosis and motor neurone disease. Palliat Med. 1998;12(5):383-94. [PMID:9924601] http://dx.doi.org/10.1191/026921698668830097

14. White CP, White MB, Russell CS. Invisible and visible symptoms of multiple sclerosis: which are more predictive of health distress? J Neurosci Nurs. 2008;40(2):85-95,102. [PMID:18481738].

15. Smith C, Hale L. The unique nature of fatigue in multiple sclerosis: prevalence, pathophysiology, contributing factors and subjective experience. Phys Ther Rev. 2007;12(1):43-51. http://dx.doi.org/10.1179/108331907X174970

16. Haskell WL, Lee IM, Pate RR, Powell KE, Blair SN, Franklin BA, Macera CA, Heath GW, Thompson PD, Bauman A; American College of Sports Medicine; American Heart Association. Physical activity and public health: updated recommendation for adults from the American College of Sports Medicine and the American Heart Association. Circulation. 2007;116(9):1081-93. [PMID:17671237] http://dx.doi.org/10.1161/CIRCULATIONAHA.107.185649

17. Garber CE, Blissmer B, Deschenes MR, Franklin BA, Lamonte MJ, Lee IM, Nieman DC, Swain DP; American College of Sports Medicine. American College of Sports Medicine position stand. Quantity and quality of exercise for developing and maintaining cardiorespiratory, musculo- skeletal, and neuromotor fitness in apparently healthy adults: guidance for prescribing exercise. Med Sci Sports Exerc. 2011;43(7):1334-59. [PMID:21694556] http://dx.doi.org/10.1249/MSS.0b013e318213fefb

18. Freeman J, Allison R. Group exercise classes in people with multiple sclerosis: a pilot study. Physiother Res Int. 2004;9(2):104-7. [PMID:15317425]

http://dx.doi.org/10.1002/pri.307

19. Oken BS, Kishiyama S, Zajdel D, Bourdette D, Carlsen J, Haas M, Hugos C, Kraemer DF, Lawrence J, Mass M. Randomized controlled trial of yoga and exercise in multiple sclerosis. Neurology. 2004;62(11):2058-64.

[PMID:15184614]

http://dx.doi.org/10.1212/01.WNL.0000129534.88602.5C

20. Wahbeh H, Elsas SM, Oken BS. Mind-body interventions: applications in neurology. Neurology. 2008;70(24):2321-28. [PMID:18541886] http://dx.doi.org/10.1212/01.wnl.0000314667.16386.5e

21. Thorne S, Con A, McGuinness L, McPherson G, Harris SR. Health care communication issues in multiple sclerosis: an interpretive description. Qual Health Res. 2004;14(1):5-22. [PMID:14725173] http://dx.doi.org/10.1177/1049732303259618

22. Kayes NM, McPherson KM, Taylor D, Schlüter PJ, Kolt GS. Facilitators and barriers to engagement in physical activity for people with multiple sclerosis: a qualitative investigation. Disabil Rehabil. 2011;33(8):625-42. [PMID:20695816] http://dx.doi.org/10.3109/09638288.2010.505992

23. Smith C, Olson K, Hale LA, Baxter D, Schneiders AG. How does fatigue influence community-based exercise participation in people with multiple sclerosis? Disabil Rehabil. 2011;33(23-24):2362-71. [PMID:21504405] http://dx.doi.org/10.3109/09638288.2011.573054

Submitted for publication January 17, 2012. Accepted in revised form October 16, 2012.

This article and any supplemental materials should be cited as:

Smith CM, Hale LA, Olson K, Baxter GD, Schneiders AG. Healthcare provider beliefs about exercise and fatigue in people with multiple sclerosis. J Rehabil Res Dev. 2013;50(5):733-44. http://dx.doi.org/10.1682/JRRD.2012.01.0012

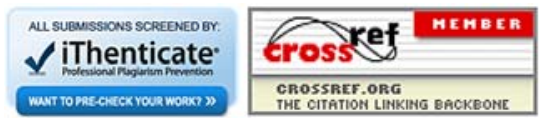


